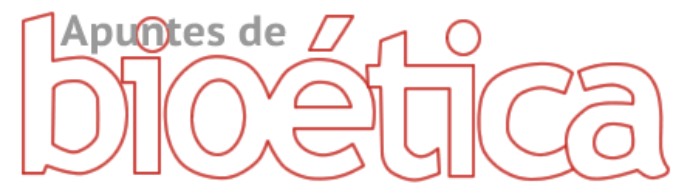

https://doi.org/10.35383/apuntes.v4i1.595 e-ISSN: $2663-4910$

Universidad Católica Santo Toribio de Mogrovejo

\title{
La vulnerabilidad humana como un valor ético en el cuidado en final de vida
}

\author{
Human vulnerability as an ethical value in end-of-life care
}

\author{
Darío Iván Radosta*, 1, a \\ diradosta@gmail.com \\ https://orcid.org/0000-0003-3507-2437
}

María del Socorro Ham ${ }^{2}$

socorroham24@gmail.com

\author{
* Autor corresponsal \\ ${ }^{1}$ CONICET-UNSAM, San Martín, Argentina. \\ 2 Hospice San Camilo, Olivos, Argentina. \\ a Doctor en Antropología Social
}

Fechas importantes

Recibido: 2021-03-14

Aceptado: 2021-05-25

Publicado online: 2021-07-26

\section{Resumen}

En este trabajo nos proponemos reflexionar acerca de cómo el carácter ontológicamente vulnerable y frágil de lo humano puede constituirse en un valor ético útil para guiar la práctica de cuidado en el final de la vida. Abordaremos en principio la distinción entre vulnerabilidad contextual y ontológica, para argumentar el carácter inherentemente frágil de la condición humana. Tomaremos la metáfora del sanador-herido como una de las fuentes a partir de las cuales la idea de vulnerabilidad se introduce como modelo de cuidado. Finalmente, expondremos algunas reflexiones acerca de cómo el cuidado en el final de la vida, propio de la filosofía hospice, permite pensar los alcances de la vulnerabilidad humana como un valor ético constitutivo de la práctica de cuidar. Entre las propuestas, creemos que la vulnerabilidad como valor ético justifica, por su carácter relacionalmente construido, el establecimiento de relaciones de ayuda simétricas y la necesidad de reconocer los propios límites.

Palabras clave: Cuidado; Hospice; Vulnerabilidad; Bioética.

\begin{abstract}
In this work we reflect on how the ontologically vulnerable and fragile character of the human can become a useful ethical value to guide the practice of care at the end-of-life. We will address in principle the distinction between contextual and ontological vulnerability, to argue the inherently fragile character of the human condition. We will take the metaphor of the wounded healer as one of the sources from which the idea of vulnerability is introduced as a model of care. Finally, we will present some reflections on how care at the end of life, typical of the hospice philosophy, allows us to
\end{abstract}


think of the scope of human vulnerability as an ethical value constitutive of the practice of caring. Among the proposals, we believe that vulnerability as an ethical value justifies, due to its relationally constructed character, the establishment of symmetrical helping relationships and the need to recognize one's own limits.

Keywords: Care; Hospice; Vulnerability; Bioethics.

\section{Introducción}

El concepto de vulnerabilidad ha sido ampliamente debatido en el campo de la bioética. Estas discusiones han mostrado por lo general las implicancias éticas que surgen en el vínculo con grupos humanos que, por diferentes motivos - entre los cuales se encuentra el padecimiento de una enfermedad que amenaza la vida - son considerados menos capaces de defender sus propios intereses (con relación, claro está, a una persona entendida hegemónicamente como "en plena capacidad" — básicamente un adulto sano -). De la necesidad de justificar un compromiso ético particular con relación a este tipo de poblaciones - que pasaron a llamarse vulnerables -, surgieron propuestas que intentaron conceptualizar la idea de vulnerabilidad. Aún con diferencias entre ellas, existe un componente que las atraviesa de manera estructural: la distinción entre la vulnerabilidad entendida como una dimensión intrínseca de lo humano - o vulnerabilidad ontológica - y como una condición que se manifiesta únicamente con relación al contexto - o vulnerabilidad contextual - (O'Neil, 1996; Goodin, 1985; Kipnis, 2003; Rogers, Mackenzie y Dodds, 2012; Kottow, 2003; Santi, 2013; Luna, 2008, 2009). Este argumento, sin embargo, fue mayormente empleado como una forma de introducir la idea de vulnerabilidad contextual en el marco del derecho, posibilitando la elaboración de documentos jurídicos dirigidos a proteger a las poblaciones humanas consideradas vulnerables (como es el caso de las Pautas CIOM-OMS o la Declaración de los Derechos del Niño). Las reflexiones filosóficas acerca de los fundamentos ontológicos de la especie tuvieron, en este sentido, un espacio mucho menor dentro de la bioética.

En este trabajo retomaremos algunas de las fuentes de reflexión en torno a la noción de vulnerabilidad, tanto desde un sentido filosófico como en la potencialidad de su aplicación práctica en el establecimiento de relaciones de acompañamiento terapéutico. A partir de allí daremos cuenta de cómo esta noción puede ser útil para pensar los fundamentos éticos del cuidado en el final de la vida. 


\section{Ser y saberse vulnerable. Vulnerabilidad ontológica y contextual}

Quisiéramos introducir, en principio, una dimensión conceptual que consideramos útil para entender en profundidad la manera en la se expresa la vulnerabilidad humana. Me refiero a la distinción hecha por Roselló (2010) entre ser y saberse vulnerable. Para este autor la vulnerabilidad se presenta como una condición intrínseca de lo humano, dada la fragilidad producto de nuestra experiencia de finitud. Como humanos, al mismo tiempo, somos conscientes de nuestra propia vulnerabilidad, en el sentido de que podemos hacernos cargo de ella. Lo que sería característico de nuestra configuración cultural occidental es una ideología que apunta a desconocer nuestra propia fragilidad ontológica, dado el valor positivo asignado a aquello que no puede ser herido. Sin embargo, existen diferentes experiencias a lo largo de la trayectoria vital de una persona que ponen de relieve esta condición: la enfermedad, y más aún cuando amenaza la propia vida, es una de ellas. La vulnerabilidad, sintetiza Roselló, se patentiza en los límites que no podemos cruzar, y nos revela el carácter precario de la existencia humana. En este sentido, y es lo que nos interesa destacar aquí, no sería necesaria una distinción entre vulnerabilidad ontológica y contextual, ya que lo que opera es siempre esa misma vulnerabilidad que es parte intrínseca de la fragilidad humana, y que, dependiendo del contexto, se nos presenta de forma más o menos patente. Somos ontológicamente vulnerables, aun cuando podamos ideológicamente desconocer ese hecho, o sea, no sabernos como tales - lo cual, para el autor, nos aleja de nuestra propia humanidad (ya que lo que compartimos como especie es justamente este carácter vulnerable) -

\section{La metáfora del sanador-herido}

Otra de las fuentes a partir de la cual es pensada la vulnerabilidad humana tiene que ver con la metáfora del sanador-herido como modelo de la relación de ayuda. Tal como marca Bermejo (2012), Carl Jung ya había utilizado este concepto (o wounded healer, en inglés) como una figura arquetípica de la relación terapéutica establecida en la consulta psicológica. El profesional, advertía, es propenso a curar a otros debido a que él mismo sostiene una herida. En la medida en que se vuelve consciente de sus propias heridas, puede establecer una conexión con las de la persona analizada, más aún en el caso de que éstas se presenten con contenidos similares. De la conciencia de la propia fragilidad surge el imperativo de curar a otros. Pese a esto, Jung no es una referencia común en el ámbito de los cuidados en el final de la vida. La metáfora del sanador-herido suele ser asociada en mayor medida con un 
libro escrito por Henri Nouwen, un sacerdote católico holandés, titulado precisamente "El sanador herido" (Nouwen, 1971). Este ensayo fue presentado por Nouwen como una guía práctica para el desempeño de la labor de ministro católico en el mundo moderno, desde un lugar de reconocimiento de la propia fragilidad. Sus reflexiones se volvieron en la actualidad un insumo fundamental para la comprensión de las relaciones de ayuda a partir de la integración de las "propias heridas" (o la propia vulnerabilidad constitutiva de nuestro ser). Bermejo (2012), por otro lado, se ha dedicado entre otras cuestiones a conceptualizar los fundamentos de la relación terapéutica empática a partir de la metáfora del sanador-herido propuesta por Nouwen. Para él, solo quien se encuentra herido, quien haya integrado a partir de un trabajo activo de reflexión su propia vulnerabilidad, podrá acompañar al otro desde ella, estableciendo así una relación no sólo empática, sino simétrica. El otro es entendido como el eco de nuestra propia vulnerabilidad, siendo la fragilidad, no solo una condición ontológica de lo humano, sino además un recurso resiliente en el proceso de curación. Tal como marca el autor, "solamente el médico herido puede curar" (2012, p.99). Podemos ver a partir de aquí cómo la introducción de la idea del sanador-herido al universo del counseling la volvió una metáfora válida para pensar la interioridad humana con relación a sus dimensiones tanto cognitivas como espirituales. Al mismo tiempo, este tipo de nociones ha tenido un impacto significativo en el desarrollo de propuestas de humanización del cuidado de la salud, con gran presencia en los cuidados en final de vida.

En la misma lógica establecida por Nouwen, existe otra fuente de referencia para el acompañamiento terapéutico basado en el modelo del sanador-herido, vinculada con la mitología griega (Bermejo, 2012; Mazzini, 2018). La historia refiere a la existencia de un centauro inmortal llamado Quirón, hijo de Filira y Cronos, a quien Hércules le infringe fortuitamente una herida con una flecha envenenada, causándole un dolor incurable. Habiendo sido formado en el arte de la medicina por sus padres adoptivos, Apolo y Atenea, Quirón decide buscar alivio a su sufrimiento, pero, y "he aquí su mítica paradoja, mientras puede curar a otros no puede curarse a sí mismo" (Bermejo, 2012, p.94). El sentido de la existencia de este personaje queda entonces ligado a sanar a los demás, movido por el dolor que le provoca su propia herida. El mito concluye con una petición de Hércules a Zeus: movido por la culpa, busca que la mortalidad de Prometeo sea cedida a Quirón, lo cual le permite fallecer para aliviar su dolor. Este acto finalmente lo asciende al cielo como la constelación de Sagitario.

Para Bermejo, es Carl Jung quien le asigna un sentido antropológico a esta historia. Quirón es para él el arquetipo del sanador-herido, dando cuenta de cómo la polaridad se constituye 
en la trama básica del mito: "el sanador lo es porque sana, pero a su vez está herido, lo cual constituye una paradoja existencial que se encarna en cada persona, tanto en la que busca curar su dolor como en la que ofrece curación" (2012, p.95). Mazzini (2018), por otro lado, da cuenta de cómo esta idea ha sido ampliamente difundida por autores que han escrito sobre el acompañamiento espiritual de los enfermos a partir de un marco de entendimiento cristiano. A partir del mito de Quirón, expresa, se puede reconocer que "quien se acerca a acompañar a otros no es un superhombre o una supermujer, alguien que desde la plena resolución de sus conflictos ilumina el camino de sus hermanos, sino un ser humano herido, conocedor del sufrimiento, del error y del pecado, quien, desde su experiencia herida, pero también de sanación en proceso, se anima a acompañar el itinerario de otras personas" (Mazzini, 2018, p. 223). Tanto los profesionales de la salud como las personas enfermas que son cuidadas poseen heridas que son propias del carácter frágil de su existencia (su condición ontológica como seres vulnerables), y que aparecen como un recurso disponible vinculado con nuestras capacidades de sanación. Nuevamente, manteniendo un esquema similar al propuesto por Bermejo, Mazzini menciona la necesidad de reconocer y cuidar nuestras propias heridas como forma de acompañar el proceso de curación de las heridas del otro. A partir de esto último podemos ver cómo se expresan ambas dimensiones de la vulnerabilidad diferenciadas por Roselló (2010). Uno es vulnerable, dada la condición frágil de lo humano y la posesión de heridas que, si bien pueden variar en contenido, todos poseemos, al mismo tiempo que uno se sabe vulnerable únicamente a partir de la relación que establece con los otros. Quienes forman parte de nuestro entorno se vuelven eco de nuestra propia vulnerabilidad. De allí la necesidad de reconocer las propias heridas, la propia fragilidad que nos identifica como humanos, para poder conectar empáticamente con la persona que tenemos al lado - y que, en el caso de estar padeciendo una enfermedad que amenaza su vida, pone de relieve con mayor fuerza el carácter precario de la existencia humana (Rosello, 2010, p.30).

\section{Cuidado y vulnerabilidad}

Dentro de la filosofía hospice - una filosofía de cuidado en el final de la vida nacida en el Reino Unido en los años sesenta (Radosta, 2019) - nos encontramos con que la vulnerabilidad es un valor fundamental que direcciona moralmente gran parte de las acciones de cuidado. La vulnerabilidad aparece aquí como una cualidad de lo humano, en el sentido de que éste comporta una fragilidad intrínseca por su condición de ser corpóreo al mismo tiempo que comprende una parte trascendente relacionada con su espiritualidad 
(Radosta, 2017). Quisiéramos reflexionar aquí acerca de la potencialidad de entender el carácter ontológicamente frágil de lo humano como un valor ético que guíe la práctica de cuidado en el final de la vida.

En principio, como vimos en los apartados anteriores, la idea de vulnerabilidad requiere de ser construida relacionalmente. Los otros son eco de la vulnerabilidad propia. Este principio es útil con el fin de establecer condiciones de simetría en la relación de cuidado, lo cual permite intentar desarticular los vínculos paternalistas comunes en el sistema biomédico (Menéndez, 2003). De la forma en que se expresa la necesidad de conectar con la vulnerabilidad del otro a partir de reconocer la propia se pueden derivar además algunas cuestiones que sirven para reflexionar éticamente sobre la práctica de cuidar en el final de la vida: como la necesidad de cuidar de quienes normalmente son reconocidos como cuidadores y el hecho de ser consciente de los límites propios en la relación de ayuda.

Esto hace que el cuidado en el final de la vida pueda ser comprendido, antes que como una relación unilineal entre quien cuida y quien es cuidado, como una red de personas que constantemente cuidan y son cuidadas. De esta idea se deriva la necesidad de cuidar de quienes comúnmente son entendidos como cuidadores. Otra de las formas en las que se expresa el cuidado desde la vulnerabilidad tiene que ver con el reconocimiento de los propios límites en la relación que se establece con el otro. Así como las personas enfermas no se encuentran enteramente compuestas por necesidades, quienes cuidan de ellas no son seres de recursos ilimitados invulnerables a cualquier tipo de estímulo, sino seres humanos ontológicamente frágiles que tienen necesidades y límites. Al estar vinculándose constantemente con situaciones de alta carga emocional (como el sufrimiento ajeno tanto de familiares como de personas enfermas por la situación que están atravesando), quienes llevan a cabo labores de cuidado en el final de la vida entienden que el cuidado también tiene que ver con el autocuidado o cuidado de uno mismo (Kearney et al, 2009; Kearney y Weininger, 2011; Benito y Rivera-Rivera, 2019; Benito et al, 2020). Como herramienta de cuidado, se entienden a sí mismos alejándose de la figura del médico que "todo lo puede" y se reconocen en cambio como seres vulnerables que deben aprender a vincularse de manera constante con los diversos límites (físicos, psíquicos, individuales o propios de la existencia humana) que surgen en su labor cotidiana. Cuidar es entonces entender los límites de cada una de las personas que cuida. Si bien algunos de estos límites tienen que ver con cuestiones prácticas (como no saber la forma correcta de realizar una tarea en específico) o emocionales (como el hecho de estar atravesando una situación de depresión o angustia), varias tienen que ver con límites que podrían ser considerados ontológicos, como el hecho 
de aprender que, frente a los problemas que padece la persona enferma o su familia, suele no existir una "solución" - ya que la muerte no es entendida necesariamente como un problema, sino como parte del flujo natural de la vida (lo cual se vincula también con una necesidad de aceptar la propia condición de ser ontológicamente vulnerable).

La expresión de la vulnerabilidad en el reconocimiento tanto de los límites propios como de las limitaciones de aquellos que nos rodean suele ser el fundamento de un imperativo moral que, vinculado al cuidado, es llevado a cabo dentro del cuidado en final de vida: trabajar en equipo. El hacer equipo suele mencionarse como una forma de llevar adelante una red de contención entre quienes suelen ser catalogados como cuidadores, con el objetivo de evitar que toda la carga del cuidado de la persona enferma y su familia recaiga sobre una sola persona. Al mismo tiempo esto permitiría, a través de la suma a de diversas experiencias y conocimientos, expandir el rango de herramientas de cuidado disponibles, extendiendo los márgenes de lo que una persona podría llevar a cabo si actuara individualmente. Esto permite sortear de alguna manera una de las críticas que se le han hecho comúnmente a la teorías éticas basadas en el cuidado: la posibilidad de opresión por parte de quienes reciben cuidados, con la posterior pérdida de la propia agencia moral de quien cuida (Luna y Salles, 2008, p. 119). El imperativo ético de cuidar en equipo, producto de la vinculación específica entre el cuidado y la vulnerabilidad como guías de la acción moral, lleva a que el cuidado de aquellos quienes regularmente son considerados como cuidadores sea tenido en cuenta en el mismo nivel de importancia que el cuidado de la persona enferma y su familia.

\section{Conclusiones}

Podemos ver cómo, entendida como un componente inherente de la condición humana, la vulnerabilidad adquiere el potencial de ser un valor ético útil en el desarrollo de prácticas de cuidado en el final de la vida. No solo se puede justificar a partir de esta noción el imperativo moral de establecer una relación de simetría con el otro - dado que se presente como el eco de mi propia vulnerabilidad - , sino que da muestras del hecho de que el otro enfermo no es ni más ni menos vulnerable por padecer una enfermedad, sino que simplemente expresa con mayor fuerza una condición inherente de lo humano, que adquiere esta fuerza en su manifestación por el hecho de que culturalmente nos desentendamos de este hecho. Al mismo tiempo, al sostenerse la idea de vulnerabilidad sobre la necesidad de reconocer los propios límites, se puede derivar de allí otro imperativo ético: el autocuidado, vinculado a su vez con la necesidad de trabajar en equipo. Los límites físico-emocionales del ser humano, 
dada su vulnerabilidad intrínseca, lo colocan en una relación de natural interdependencia con el resto de las personas. Esto nos ayuda a entender el cuidado, y más aún el cuidado en el final de la vida, como un conjunto de relaciones multidireccionadas, antes que como una relación unilineal entre alguien que cuida y alguien que es cuidado.

\section{Referencias}

Benito, E. y Rivera-Rivera, P. (2019). El cultivo de la autoconciencia y el bienestar emocional en los profesionales que trabajan con el sufrimiento. RIECS, 4(S1), 2530-2787.

Benito, E., Rivera-Rivera, P., Yaeguer, J. y Specos, M. (2020). Presencia, autoconciencia y autocuidado de los profesionales que trabajan con el sufrimiento. Apuntes de bioética, 3(1), $72-88$

Bermejo, J. (2012). Empatía terapéutica. La compasión del sanador herido. Bilbao: Desclée de Brouver.

Goodin, R. (1985). Protecting the Vulnerable. Chicago: The University of Chicago Press.

Kearney, M. et al (2009), Self-care of Physicians Caring for Patients at the End of Life. Perspectives on care at the close of life. 301(11), 1155-1164.

Kearney, M. y Weininger, R. (2011). Whole person self-care: self-care from the inside out. Springer. New York, 109-125.

Kipnis, K. (2003), Seven Vulnerabilities in the pediatric research subject. Theoretical Medicine. 24, 107-120.

Kottow, M. (2003). The vulnerable and the susceptible. Bioethics. 17(5-6), 460-471.

Luna, F. (2008). Vulnerabilidad: la metáfora de las capas, Jurisprudencia Argentina, 4 (1). Pp. 60-67.

Luna, F. (2009). Elucidating the Concept of Vulnerability. Layers not Labels. IJFAB, 2(1).

Luna, F. Y Salles, A. (2008). Bioética: nuevas reflexiones sobre debates clásicos. Buenos Aires: Fondo de Cultura Económica.

Mazzini, M. (2018). El cuidado hospice como acompañamiento espiritual. Teología urbana. Prácticas de espiritualidad popular. Azcuy, V. (coord.). Buenos Aires: Ágape Libros.

Nouwen, H. (1971). El sanador herido. PPC. Madrid.

O’Neill, O. (1996). Towards Justice and Virtue. Cambridge: Cambridge University Press.

Radosta, D. (2017). Devolverle al enfermo su entidad de persona. El cuidado hospice como dispositivo terapéutico. Plural. Revista de la Asociación Latinoamericana de Antropología.

Radosta, D. (2019). Reconstrucción histórica del surgimiento del moderno movimiento hospice. Scripta Ethnologica. Vol. XLI, 9-41.

Rogers, W., Mackenzie, C. y Dodds, S. (2012). Why Bioethics Needs a Concept of Vulnerability. IJFAB, 5(2), 11-38.

Roselló, F. (2010). Hacia una antropología de la vulnerabilidad. Revista Forma. Vol 2, 25-32. 
Santi, F. (2013). Aportes para una nueva concepción de la vulnerabilidad en ética de la investigación.

Fundación Jaime Roca. Premio anual de bioética 2013.

\section{Cómo citar este trabajo}

Radosta, D. I., \& Ham, M. del S. (2021). La vulnerabilidad humana como un valor ético en el cuidado en final de vida. Apuntes De Bioética, 4(1), 23-31. https://doi.org/10.35383/apuntes.v4i1.595

\section{Financiación}

El presente artículo no cuenta con financiación específica para su desarrollo y/o publicación.

\section{Conflicto de interés}

Los autores del artículo declaran no tener ningún conflicto de intereses en su realización.

(c) Los autores. Este artículo es publicado por la Revista Apuntes de Bioética del Instituto de Bioética, Universidad Católica Santo Toribio de Mogrovejo. que permite el uso no comercial, distribución y reproducción en cualquier medio, siempre que la obra original sea debidamente citada. 